\title{
Using Pathological Elements in Analog Circuit Analysis and Simulation
}

\author{
$1^{\text {st }}$ Alexandru GRIB \\ PhD Student, Faculty of Electrical \\ Engineering Faculty, University \\ POLITEHNICA of Bucharest, \\ radugrib@hotmail.com
}

$4^{\text {th }}$ Alina CONSTANTIN

PhD Student, Faculty of Electrical Engineering Faculty, University

POLITEHNICA of Bucharest,

Romania, e-mail: alinac4u@gmail.com

\author{
$2^{\text {nd }}$ Zoltan ERDEI \\ PhD Associated Prof., Faculty of \\ Electrical Engineering Faculty, \\ Technical University of Cluj Napoca, \\ Zoltan.Erdei@cunbm.utcluj.ro \\ $5^{\text {th }}$ Mihai IORDACHE \\ Prof., Faculty of Electrical Engineering \\ Faculty, University POLITEHNICA of \\ Bucharest, \\ mihai.iordache@upb.ro
}

\author{
$3^{\text {rd }}$ Mihaela Cristina TURCU \\ $\mathrm{PhD}$ Student, Faculty of Electrical \\ Engineering Faculty, University \\ POLITEHNICA of Bucharest, \\ turcu_mihaela2003@yahoo.com
}

\begin{abstract}
This paper is focused on some kind of mystery circuit nullator, nullor, current mirror, and voltage mirror, all of them known as pathological elements. These pathological elements can be used to perform circuit modelling, symbolic circuit analysis, circuit synthesis, circuit design and to develop applications that involve modern active devices. It is described a new modeling of nullor-based active devices from the circuit abstraction level. In this paper it is presented the way all types of equations which describe the circuit containing nullors can be directly formulated from the diagrams of these circuits if we took into account that a nullator is an over-determined two-port circuit element (zero voltage, zero current) and the norator is an undetermined two-port circuit element (any voltage and any current). To simulate the nullors with ideal voltage controlled voltage sources, ec $=A c \_c u_{c}$, with the control gate an ideal independent current source, $j_{C}=0.0 \mathrm{~A}$ and with the amplification (transfer) factor $A_{c_{-} C}$ very big (theoretically $\infty)$, the analog circuits with nullors can be analyzed by using any of the existing simulation software. By this way, it was possible the elaboration of efficient algorithms for an automatic formulation of Kirchhoff's equations, of loop equations, of modified nodal equations and of state equations for circuits with pathological elements. These procedures can be easily implemented in dedicated programs for the simulations of the complex analog circuits with pathological elements. The example presented in this paper validates the presented models for nullors.
\end{abstract}

Keywords-component, formatting, style, styling, insert (key words)

\section{INTRODUCTION}

Usually, the active devices are described through equivalent circuits that contain voltage-controlled voltage sources (VCVS), voltage-controlled current sources (VCCS), current-controlled voltage sources (CCVS) or currentcontrolled current sources (CCCS). These ones are very useful for the circuits design based on specialized software (e.g. SPICE). Using this kind of software applications, there can be built, with a high accuracy, many types of active circuit devices [1 - 24]. Also, these equivalent circuits are used to elaborate the analog circuit's equations systems based on various methods, like Nodal Analysis Method (NAM), Modified Nodal Analysis (MNA) Method, State Variable Method (SVM) and Tableau Method (TM).

It has been demonstrated that, according to the symbolic analysis principles, the Nodal Analysis Method (NAM) is restrictive because the admittance matrix must contain only the elements compatible with the Nodal Analysis (NA). This problem can be easily resolved through the Modified Nodal Analysis Method (MNAM), adding one row and one column for each element which is not compatible with the classic Nodal Analysis Method, [20 - 24]. One of the problems generated by this kind of approach is related to the size of the admittance matrix. This matrix will become bigger, according to the structure of the circuit and types of its elements.

Concerning the models to be used in the analog circuit analysis, the requirement of a high accuracy could lead to complicated calculations and this is way compact models are preferred during the analysis of circuits, mainly for the use of much more simple equations, [1 - 12]. These models are more effective for the optimization of the modeling and simulation time during the analysis process. From this point of view, the pathological elements (nullator, nullor, current mirror, and voltage mirror etc) proved already its efficiency in the active devices modeling. Also, in the models based on the nullor element, all the parasitic elements can be included to analyze their contribution to the analog circuit response [20 23]. The nullors are very useful for the analog circuits modeling because the circuit topology can be represented with two-terminal components, like resistors, capacitors, nullators, norators and independent voltage and/or current sources. Also, it can be highlighted that all controlled sources can be represented with equivalent circuits using nullor elements, [11 - 14]. Therefore, the equations system, for the equivalent circuit based on nullor elements, will be developed in accordance with the classic Nodal Analysis Method. The nullor will be one of the basic components for the active devices models, considering that the model should be developed in the simplest manner and the accuracy of the circuit behavior simulation must be in acceptable limits, $[6,11$ $-16]$. According to this approach, this chapter will show the 
problems regarding the small-signal models of the active devices that have been developed with nullors.

The norator can be defined as an ideal circuit with two terminals (Fig. 1a), which is characterized by random values for the current $(i)$ and voltage $(v)$ at the terminals. With other words, the norator does not have any defined relationship. The current and the voltage have values that are affected only by the external circuit which controls the norator.

The nullator can be defined as an ideal circuit with two terminals, which is characterized by null values for the current and voltage, at the terminals. The symbol used for its graphic representation is shown in Fig. 1b. For this kind of circuits, there can be defined two relationships.

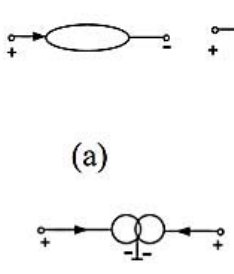

(d)

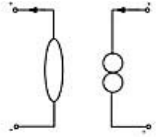

(c) (b)

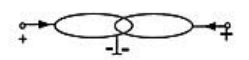

(e)
Figure 1. a) Nullator symbol; b) Norator symbol; c) Nullor symbol; d) Current mirror; e) Voltage mirror,

$$
[1,2,17] \text {. }
$$

A nullator and a norator together form a normal twoport circuit called nullor (Fig. 1c), which has the number of definition relationship equal to the number of gates.

The nullor is a two-port circuit that comprises a nullator and a norator. The number of definition relationships for this circuit is the same with the number of its gates. Also, this kind of circuits can be assimilated to an ideal operational amplifier for which the input (voltage and current) is null and the output gate delivers unknown output voltage and current values resulted from the inputs figures multiplied by the gain/transfer factor, which is infinite (Fig. 2).
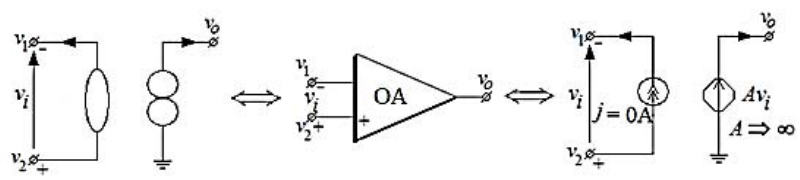

Figure 2. Nullor simulation by an ideal operational amplifier.

Theoretically, a nullor is just a diport circuit that comprises a nullator as input and a norator as output (Fig. 1c). The transferred parameters have zero values and the circuit input-output dependency is described by the circuit matrix equation.

The linear/linearized circuit analysis and development have been performed using the nullator and norator as theoretical active devices, $[1-13]$. Tellegen has been the first who presented the ideal operational amplifier theory and later, in 1964, Carlin tried to consider nullators and norators as single active devices in the circuit analysis, $[3,4]$. He thought that these active devices cannot build physically. Tellegen also took in consideration that these devices must be seen only as mathematical models without any physical support. Again, Carlin proposed the combination of the nullator and the norator, which resulted in a useful physical device, the nullor [4 - 23].

In $[12,13]$ the behavior of the nullators, norators and nullors from the point of view of the voltage, respectively of the current, in Gv - the voltage graph and, respectively Gi - the current graph is presented.

The input port of the nullor is modeled by the nullator which is characterized by two equations:

$$
v_{1}=v_{2}=\text { arbitrary, } i_{1}=i_{2}=0 .
$$

So, the nullator is simultaneously one open in $\mathrm{G}_{\mathrm{i}}$ current graph and short-circuit in $\mathrm{G}_{\mathrm{v}}$ voltage graph. The output port of the nullor is modeled by the norator where both, the voltage and current can be assumed to have arbitrary values:

$$
v_{1} \neq v_{2}=\text { arbitrary, } i_{1}=i_{2}=\text { arbitrary }
$$

The nullor is a two-port element and it is known as universal active element [13, 22, 23]. This concept means that the nullor along with capacitors and resistors can be used to design a maximum number of functions with the minimum number of active devices. That is, if a suitable set of linear and nonlinear passive elements is available, then no active element other than nullors are needed to implement any linear or nonlinear circuit function. So, nullators, norators, resistances, along with capacitances can synthesize a complete set of linear or linearized equations.

\section{NULLOR EQUIVALENCES}

From the beginning, the nullor circuit has been considered very efficient for the analog circuit analysis, modeling and synthesis. Therefore, there are many records regarding methods and algorithms based on nullor circuits, used for the active devices analysis and modeling, [3 - 23].

Because any analog network can be modeled with nullators, norators and impedances, it is worth mentioning some equivalence between them, since they can be very useful in the synthesis and modeling process. Thus, the main equivalences of the combinations between nullators and norators for networks that contain nullors and impedances are shown in Fig. 3. For instance, in Fig. 3a, a current flowing from node $a$, cannot flow from $a$ to $b$, since the current through the nullator is zero, so that a series connection of the nullator and norator is equivalent to one open-circuit. In Fig. $3 \mathrm{~b}$, the current can flow from $a$ to $b$ through the norator, also the voltage across $a$ and $b$ becomes zero from the property of the nullator, so that a parallel connection of the nullator and norator is equivalent to one short-circuit. The remaining connections have equivalents according to the nullator and norator $i-v$ characteristics. 


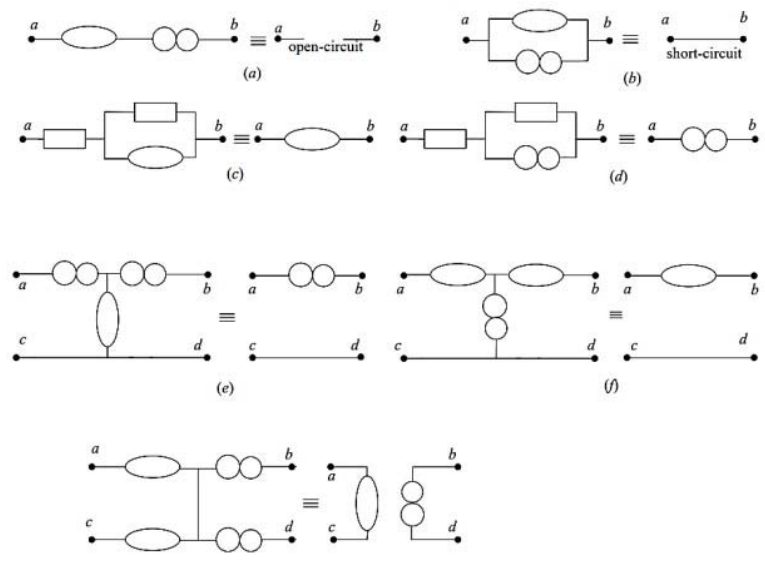

Figure 3. Nullator and norator equivalences $[1,2,17]$.

Another approach, the nullor along with grounded resistors can be manipulated in order to obtain inverting properties, features that the nullator and the norator cannot model by themselves $[22,23]$. The main purpose of the introduction of inverting properties is that the behavior of some active devices involves inverting the voltage and current input-signals. In this sense, the Current-Mirror (CM) and the Voltage- Mirror (VM), both as active devices, can perform this task and their behavior also should be modeled with the nullor. Thus, by manipulating the nullor along with grounded resistors, the behavior of a CM and a VM, both with ideal unity-gain can easily be obtained, as shown in Fig. 4 [23].

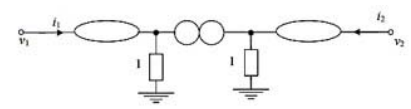

(a)

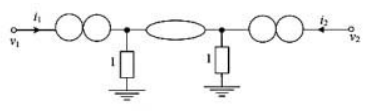

(b)
Figure 4. Nullor and grounded resistor-based VM (a) and CM (b), $[1,2,17]$.

Therefore, by analyzing the equivalent circuits, one can see that the VM, shown in Fig. 4a, is characterized by:

$$
v_{2}=-v_{1}=\text { arbitrary, } i_{1}=i_{2}=0 .
$$

and the CM, shown in Fig. 4, b, is characterized by:

$$
v_{2} \neq v_{1}=\text { arbitrary, } i_{1}=i_{2}=\text { arbitrary. }
$$

At the end, the inverting behavior of the nullator and norator is achieved. In [4 - 8], the nullor - based models of the VM and CM include parasitic elements. In the same manner as for the nullor, equivalences between the combinations of nullators, norators, CMs, VMs and impedances within an analog network can be obtained. Note, however, if the v1- or v2-terminal from Fig. 3, a is grounded and by applying the equivalences shown in Fig. 1, the VM is reduced to a nullator. In the same manner, if any terminal in Fig. $3 \mathrm{~b}$ is grounded and after by applying the equivalences shown in Fig. 2, then a norator is obtained.

Using the nullor, the ideal model of the bipolar junction transistor (BJT) was introduced in $[1,2,10]$, where the base (B), collector (C) and emitter (E) terminals are denoted as shown in Fig. 5a. As one sees, the voltage between $\mathrm{BE}$ is the sum of the voltage across the nullator and the resistor, however, since the voltage across the nullator beings zero, then the input voltage drops across terminals A, B where a resistor is connected. Also, since the current through the nullator beings zero, then the current through the resistor goes to the output through the collector. In a similar way, nowadays the model in Fig. 5a can be used to describe the MOSFET whose terminals are gate $(G)$, drain $(D)$ and source $(S)$. In both models for the BJT and MOSFET, the output current is proportional to the inverse of the resistance value, which for amplifier design it is known as the transconductance denoted by gm. In an extended version like the topology shown in Fig. $5 \mathrm{~b}$, the output is also a current that is proportional to the transconductance gm in which the input voltage drops because the voltage across the nullators being zero, so that $i_{o}=g_{m} v_{i n}$ and the direction is imposed by the polarities of the nullators, i.e. the negative input goes to terminal $\mathrm{A}$ and the positive input goes to $\mathrm{B}$, so that the polarity of the transconductance gm goes from $\mathrm{B}(+)$ to $\mathrm{A}(-)$, as denoted by the arrows in the norators at the output port, and as shown in Fig. 5b. It is also possible to joint a nullator-norator pair to model a negative-type secondgeneration current conveyor, as shown in Fig. 5c, where the equations $v_{x}=v_{y}, i_{y}=0$ and $i_{z}=-i_{x}$ are accomplished. These models will be used in the next sections to show how to perform symbolic analysis and synthesis of nullor and pathological mirror networks.

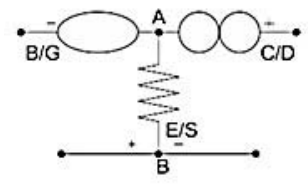

(a)

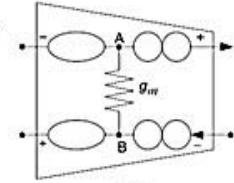

(b)

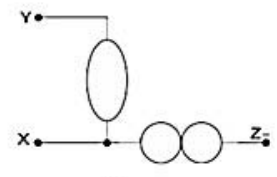

(c)
Figure 5. Ideal model of: a) The bipolar junction transistor (BJT) (or metal-oxide-semiconductor field-effect-transistor (MOSFET)), b) The operational transconductance amplifier (OTA), and c) Negative-type second-generation current conveyor (CCII-), [1, 2, 17].

Starting from the equivalent schemas with nullors, $[11,12]$, the parameters associated with the controlled and the controlling branches are presented in [12]. It must be noticed that in circuits with nullors, the dependent sources can remain in circuit without being replaced by equivalent diagrams containing nullors (see example).

\section{Formulation OF THE EQUATIONS FOR THE ANALOG CIRCUITS WITH NULLORS}

The controlled sources can be equivalent, [11 - 14], with equivalent schemes which contain nullors and passive two-port circuit elements. $G^{i}$ graph it is used to write the Kirchhoff current law, while $G^{v}$ graph is used for the Kirchhoff voltage law, [12]. The constitutive equations of all circuit elements are written as relationships between the $G^{i}$ currents and $G^{v}$ voltages. The use of the two graphs is very useful for automatic generation of the generalized topological formula with homogenous parameters, valid for any transfer function of a lumped, linear and time-invariant circuit, [11$14]$.

Now, there are several commercial and professional software families for electric circuit numerical simulation, (SPICE, LTSPICE, CADENCE, SABER, CASPOC, PHOENIX, SIMULINK, DIANA, EMTP, TACS, KREAN, PETS), [19, 20]. Some of them have versions dedicated for education in electrical engineering. But no specialized software can cover all the situations that can occur in practice. A major disadvantage of the professional and commercial 
software is that the user capacity to adjust the software is very limited. Therefore, the computation of some problems that may occur in practice requires the necessity of the development or programs oriented towards the specific application or category of applications. This is the reason for which the authors have developed the following software products [21, 22]: SCAP - Symbolic Circuit Analysis Program, SYTFG - SYmbolic Transfer Function Generation SYSEG - SYmbolic State Equation Generation) and SSEMG - Semi-State Equation Matrix Generation.

By replacing the nullors form the equivalent scheme from figure 2 , the circuits with nullors can be simulated with the above-mentioned programs, without being necessary the generation of the current and voltage graphs.

As follows, we present different circuits with nullors considering the behavior of the nullors from the current and voltage point of view and using the equivalent diagram from figure 2 .

Kirchhoff's equations, loop equations, modified nodal equations and the state equations can be manually formulated, for circuits with nullors, in a direct manner without replacing the nullors with the equivalent schemes from figure 2. These equations cannot be automatically generated, by using the existing software, because these programs do not admit as primitive circuit elements the nullors. To use these programs, to simulate the circuits with nullors, these should be replaced with the equivalent scheme from figure 2 .

\section{EXAMPLE}

Let us consider a Miller amplifier as presented in figure $6 \mathrm{a},[1,2]$. By replacing the MOSFET transistors by the equivalent scheme with nullors, $[1,2]$, the circuit from figure $6 \mathrm{~b}$ is obtained.

We consider, for each nullor in Fig. 6b, the equivalent circuit from Fig. 2, and the following numeric values of the circuit parameters: $A=\infty, g_{m 1}=0.0001 \mathrm{~S} ; g_{m 8}$ $=0.0002 \mathrm{~S} ; R=10000.0 \Omega ; R_{\text {Aip }}=2000.0 \Omega ; g_{o p}=0.0005 \mathrm{~S}$; $g_{\text {om }}=0.0002 \mathrm{~S} ; C_{m}=1.0 \mathrm{e}-12 \mathrm{~F} ; C_{L}=4.0 \mathrm{e}-12 \mathrm{~F} ; g_{o 8}=0.0005$ $\mathrm{S} ; g_{o n 8}=0.0002 \mathrm{~S} ; g_{o n 6}=0.0002 \mathrm{~S} ; g_{o 8}=0.0005 \mathrm{~S} ; g_{o p}=$ $0.0002 \mathrm{~S} ; C_{g s 1}=2.0 \mathrm{e}-12 \mathrm{~F}$ şi $C_{\mathrm{gs} 2}=5.0 \mathrm{e}-12 \mathrm{~F}$.

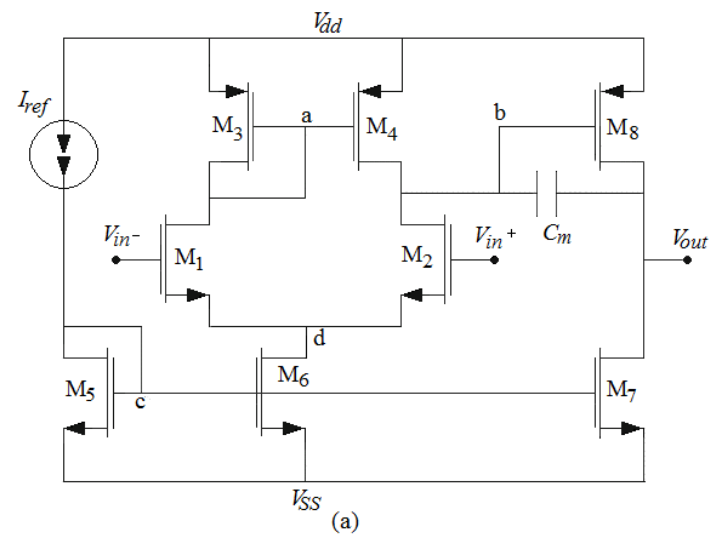

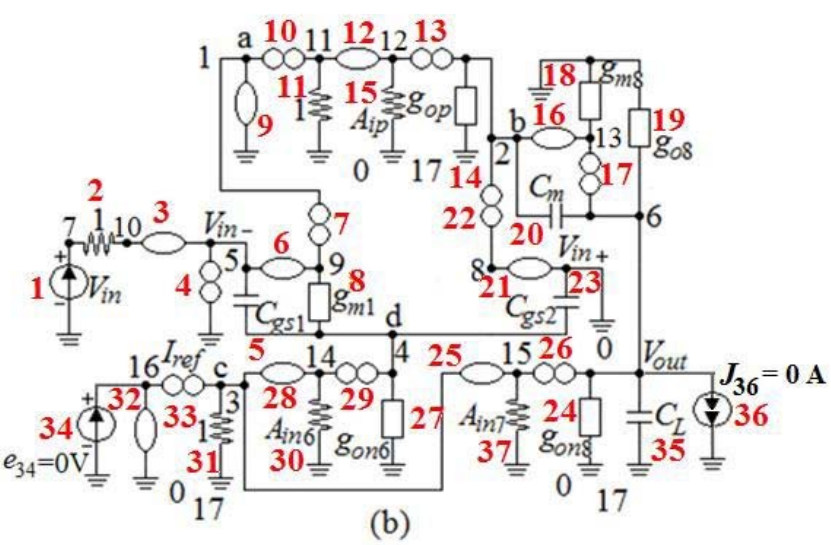

Figure 6. Miller amplifier: a) MOSFET circuit, [1, 2]; b) Its nullor equivalent taken from [17].

Analyzing the circuit in Fig. 6b by CSAP program $[16,17]$, the following results are obtained:

1. Voltage gains:

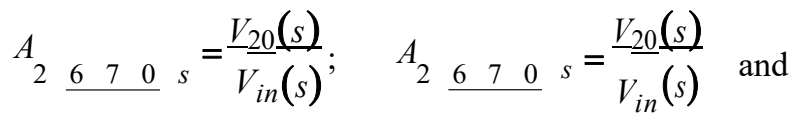

$$
\begin{aligned}
& A_{2} 6 \quad 7 \quad s=\frac{V_{17}(s)}{V_{\text {in }}(s)} \text { : } \\
& \text { A6_0_7_0_s: }=\frac{\left.0.00050(-0.410-0.8010 \quad s+0.5010 s)^{-7}\right)^{-232}}{\left(0.7010^{-11} s+0.0003\right)\left(0.1410^{-6}+0.4010^{-23} s^{2}+0.1910^{-14} s\right)}
\end{aligned}
$$

(5)

$$
\text { A2_6_7_0_s: }=\frac{0.00050\left(0.1810^{-6}+0.5310^{-14} s+0.2010^{-22} s^{2}\right)}{\left(0.7010^{-11} s+0.0003\right)\left(0.1410^{-6}+0.4010^{-23} s^{2}+0.1910^{-14} s\right)}
$$

A13_6_7_0 $s:=\frac{0.00050\left(0.1810^{-6}+0.5310^{-14} s+0.2010-22 s^{2}\right)}{(0.7010 s+0.0003)(0.1410+0.4010 s+0.1910 s)}$

2. Poles of the three voltage gains:

Poles_A6_O_7_O: $=\left[-0.4310^{8},-0.9110^{8},-0.3810^{9}\right]$

Poles_A2_6_7_0: $=\left[-0.4310^{8},-0.9110^{8},-0.3810^{9}\right]$

Poles_A13_6_7_0: $=\left[-0.4310^{8},-0.9110^{8},-0.3810^{9}\right]$.

We note that the poles of the three voltage gains are identically.

The Bode diagrams for the voltage transfer factors

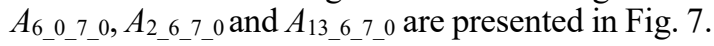




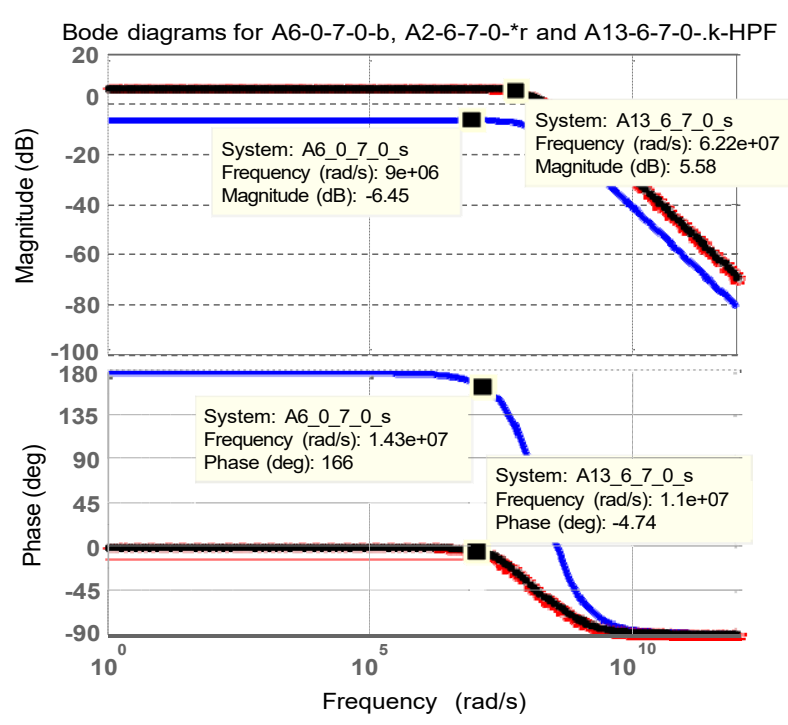

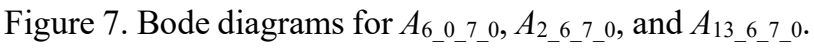

From Fig. 7 we can note that the Bode diagrams for $A_{2 \_6 \_7 \_0}$ and $A_{13 \_6 \_70}$ are identically, according to their above expressions.

In Fig. 8 are shown the distributions, in the $s$ plan, of the poles and zeros for $A_{2 \_6 \_70}$ and $A_{13 \_6}$ __ 0 .

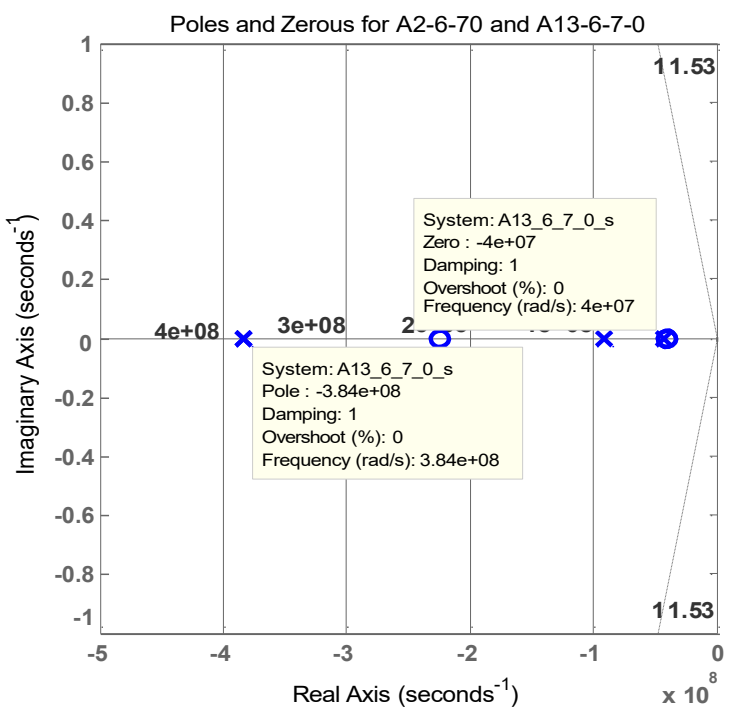

Figure 8. Poles and Zerous for A2_6_7_0 and A13_6_7_0.

\section{CONCLUSIONS}

Our paper presented a new modeling of nullor-based active devices from the circuit level of abstraction. After a brief overview on the nullor concept and its properties, the modeling of active devices not only at the voltage-mode but also at the current-mode and the mixed-mode of operation from two-port and four-terminal network point of view are described in some details.

In general, for the simulation of the four nullorcontrolled sources and, in general, for the modeling of multiport circuit elements with equivalent circuits consisting of bipolar circuit elements and the nullor behavior from current and voltage point of view, for a systematic formulation of $\mathrm{KCL}$, one has to associate to the analyzed circuit, the current graph $G^{i}$ and, respectively a voltage graph $G^{v}$ in order to formulate the KVL. The use of the two graphs in analog circuit analysis with nullors implies the elaboration of efficient algorithms for an automatic generation of these graphs and their implementation in specialized software.

To simulate the nullors with ideal voltage controlled voltage sources, $e_{c}=A_{c_{-} C} u_{\mathrm{C}}$, with the control gate an ideal independent current source, $j_{C}=0.0 \mathrm{~A}$ and with the amplification (transfer) factor $A_{c_{-}}$very big (theoretically $\infty$ ), the analog circuits with nullors can be analyzed by using any of the existing simulation software.

Using the equivalent circuit in Fig. 2 for the nullors, we could presented in this paper, the way in which all types of equations which describe the circuit working with nullors can be directly formulated from the diagrams of these circuits if we took into account that a nullator is an over-determined twoport circuit element (zero voltage, zero current) and the norator is an undetermined two-port circuit element (any voltage and any current). Therefore, all types of equations, which describe the circuits working, can be formulated in a simple manner, no matter if the analyzed circuit contains nullors or not. In this way, it was possible the elaboration of efficient algorithms for an automatic formulation of Kirchhoff's equations, of loop equations, of modified nodal equations and of state equations for circuits with nullors. These procedures can be easily implemented in dedicated programs for the simulations of the complex analog circuits with nullors. The example presented in detail validates the presented models for nullors.

\section{ACKNOWLEDGMENT}

The work has been funded by the Operational Programme Human Capital of the Ministry of European Funds through the Financial Agreement 51675/09.07.2019, SMIS code

\section{5 .}

\section{REFERENCES}

[1]. Mourad Fakhfakh, Marian Pierzchala - Editors, Pathological Elements in Analog Circuit Design, Lecture Notes in Electrical Engineering 479, ISBN 978-3-319-75156-6 ISBN 978-3-319-75157-3 (eBook), https://doi.org/10.1007/978-3-319-75157-3, Springer Customer Service Center, 2019.

[2]. Esteban Tlelo - Cuautle - Editor, Advances in Analog Circuits, Published by InTech Janeza Trdine 9, 51000 Rijeka, Croatia, ISBN 978-953-307-323-1, Free online editions of In Tech Books and Journals can be found at www.intechopen.com, 2011.

[3]. Răzvan Asanache, Mihai Iordache, Dragos Niculae, Marilena Stanculescu, Lavinia Bobaru, Victor Bucata, Sorin Deleanu, "On Circuit Analysis and Simulation of Networks with Nullors", Proceedings of 
the The $8^{\text {th }}$ International Conference on Modern Power Systems (MPS 2019), 21-23 May 2019, ClujNapoca, Romania, IEEE Xplore, INSPEC Accession Number:17028441, IEEE, DOI:10.1109/MPS.2019.8759698

[4]. R. Hashemianm, "Nullors in amplifier design for bandwidth using multiple feedbacks", In: IEEE 30th Canadian conference on electrical and computer engineering (CCECE), Windsor, CA, 30 April- 3 May, 2017.

[5]. R. Hashemian R, “Application of nullors in designing analog circuits for bandwidth", In: Proceedings of the IEEE International Conference on Electro/Information Technology, EIT2016, Grand Forks, ND, pp. 19-21, May 2016.

[6]. R. Hashemian R, "Fixator-norator pairs versus direct analytical tools in performing analog circuit designs", IEEE Trans Circuits Syst II Exp Briefs 61(8):569$573,2014$.

[7]. A. S. Sedra, K. , C. Smith KC, Microelectronic circuits, 7th edn. Oxford University Press, New York, 2014

[8]. R. J. Baker RJ, CMOS, Circuit Design, Layout, and Simulation, 2nd edn. IEEE Press, Wiley Interscience, 2008, pp 613-823

[9]. Q. Yu, C. Sechen, "A Unified Approach to the Approximation Symbolic Analysis of Large Analog Integrated Circuits", IEEE Trans. on Circuits and Systems - I: Fundamental Theory and Applications, Vol. 43, 8, 1996, pp.656-669.

[10]. H.J. Carlin, "Singular networks elements", IEEE Transactions on Circuit Theory, vol. 11, pp. 67-72, March 1964.

[11]. B.D.H. Tellegen, "On nullators and norators", IEEE Transactions on Circuit Theory, vol. CT-13, pp. 466469, December 1966.

[12]. A. Carlosena and G. S. Moschytz, "Nullators and norators in voltage to current mode transformations", International Journal of Circuit Theory and Applications, vol. 21, pp. 421- 424, 1993.

[13]. E. Tlelo-Cuautle, C. Sánchez-López, E. MartínezRomero, S. X.-D. Tan, "Symbolic analysis of analog circuits containing voltage mirrors and current mirrors", Analog Integrated Circuits and Signal Processing, [Online] DOI: 10.1007/s10470-010-9455y, February 2010.

[14]. E. Tlelo-Cuautle, E. Martinez-Romero, C. SánchezLópez, and S. X.-D. Tan, "Symbolic formulation method for mixed-mode analog circuits using nullors," in IEEE International Conference on Electronics, Circuits and Systems, 2009, pp. 856-859.

[15]. G. Gielen, W. Sansen, Symbolic Analysis for Automated Design of Analog Integrated Circuits, Boston, MA: Kluwer Academic, 1991.
[16]. M. Iordache, "Generalization of the topological formulas with homogeneous parameters", Rev. Roum. Sci. Techn. - Électrotechn. et Énerg., 4, 1980, pp. 501513.

[17]. Mihai Iordache, Lucia Dumitriu, Dragoș Niculae, Marilena Stănculescu, Victor Bucată, Georgiana Rezmeriță, Chapter 4 "Circuit Analyses with Nullors", Book chapter in the book, Pathological Elements in Analog Circuit Design, Mourad Fakhfakh, Marian Pierzchala - Editors, Lecture Notes in Electrical Engineering 479, ISBN 978-3-319-75156-6 ISBN 978-3-319-75157-3

(eBook) https://doi.org/10.1007/978-3-319-75157-3, Springer Customer Service Center

[18]. W. Verhagen, G. Gielen, "An efficient evaluation scheme for linear transfer functions using the determinant decision diagram representation of the system determinant", Proceedings of the Fifth International Workshop on Symbolic Methods and applications in Circuit Design, SMACD'98, Kaiserslautern, Germany, October 8-9, 1998, pp. 99105.

[19]. J. D. Rodriguez-Garcia, O. Guerra, E. Roca, F. V. Fernandez and A. R. Vazquez, "A New Simplification Before and During Generation Algorithm", Proceedings of the Fifth International Workshop on Symbolic Methods and Applications in Circuit Design, SMACD'98, Kaiserslautern, Germany, October 8-9, 1998, pp. 110-124.

[20]. M. Galan, F.V. Fernandez and A. R. Vazquez, "Comparison of Matroid Intersection Algorithms for Large Circuit Analysis", prceedings of the IEEE International Symposium on Circuits and Systems, ISCAS'97, Hong Kong, June 9-12, 1997, pp. 17841787.

[21]. M. Iordache, Lucia Dumitriu, Computer-Aided Simulation of Analogue Circuits: Algorithms and Computational Techniques, POLITEHNICA Press Publishing, Bucharest, 2014, in ROM.

[22]. M. Iordache, Symbolic, Numeric - Symbolic and Numeric Simulation of Analog Circuits - User Guides, MATRIX ROM Publishing, Bucharest, 2015, in $\mathrm{ROM}$

[23]. M. Fakhfakh, E, Tlelo-Cuautle and F. V. Fernandez (Eds), Design of Analog Circuits through Symbolic Analysis, Bentham Science Publishers, 2012, pp. 83114, pp. 228-262 respectively (e-book), DOI: 10.2174/97816080509561120101, eISBN: 978160805-095-6.

[24]. F.V. Fernández, A. Rodriguez-Vázquez, J.L. Huertas, and G. GiFelen, Symbolic analysis techniques: applications to analog design automation, Piscataway, NJ: IEEE Press, 1998. 